\title{
DIABETIC RETINOPATHY. ASSOCIATION WITH METABOLIC COMPENSATION, DURATION OF DIABETES AND OTHER MICRO AND MACROVASCULAR COMPLICATIONS IN PATIENTS WITH TYPE 1 DIABETES MELLITUS
}

\author{
Dr. Lelde Ullase, ${ }^{* * *}$, Kristīne Ducena**, Dr. Dace Markevica***, prof. Guna Laganovska*** \\ * Resident in Ophthalmology, Faculty of Medicine, University of Latvia Riga, Latvia \\ ** SIA University of Latvia Medical postgraduate education institute, Riga, Latvia \\ *** Department of Ophthalmology, Pauls Stradins Clinical University Hospital, Riga, Latvia
}

\begin{abstract}
Summary
Introduction. Diabetic retinopathy (DR) is a severe complication that can lead to complete vision loss and still is one of the main blindness-causing reasons among patients with type 1 diabetes mellitus (T1DM). DR as a complication can cause vision loss to people at their working age. More than $90 \%$ of patients with type 1 diabetes will develop DR by 20 years post diagnosis (Leslie R.Dye, 2018). DR is more likely to develop in patients with T1DM (Kanski's Clinical Ophthalmology, 2016). This complication can be very serious speaking of the ability to see. Sometimes vitrectomy plays a vital role in the management of severe complications of DR at its end-stage (Myron Yanoff et al., 2020).

Aim of the study. To prove the development severity of DR that depends on the duration of diabetes and metabolic compensation. Additionally, to determine retinopathy's association with other micro and macrovascular diabetes mellitus complications for a better understanding of what are the contributory factors for these complications to develop and which of those may coexist.

Materials and Methods. A retrospective study was held at the Pauls Stradins Clinical University Hospital (Riga, Latvia). From January 2016 to March 2018, 79 (158 eyes) patient histories were analyzed who have type 1 diabetes mellitus. To obtain more precise research results, almost all patients have done check-up visits to one certain ophthalmologist. The IBM SPSS Statistics version 25.0 was used to process data. Tables were made in SPSS and Microsoft Excel 2016 programs. Statistically significant value (p) was set at $<0,05$.

Results. No statistically significant difference is seen in the mean duration of the disease: in the group of proliferative diabetic retinopathy $(P D R)$ : 25.23 (median $=22.0$ ) years and non-proliferative group: 24.68 (median $=23.50$ ) years. Results show that the duration of diabetes mellitus is considerably smaller in a group without DR 11.24 (median $=8.50$ ) years. Metabolic compensation $(\%)$ in diabetes mellitus is not statistically different between patients with diverse forms of DR; no association found either. No statistically significant difference in best corrected visual acuity (BCVA) was detected among patients with various forms of DR. Three groups were compared: both types of DR and no DR. It was detected that BCVA in patients without DR was higher in both eyes: $0.83 \pm 0.27$ dioptres. No statistically significant difference $\left(p_{x}>0.05\right)$ was detected between the groups of $D R$ and therefore no association was made between the form/existence of DR and arterial hypertension. There is a strong association between DR and microvascular complications $(V=0.40)$ with the existence of $D R$ and there is an even stronger association $(V=0.61)$ with the forms of DR. There is no statistically reliable difference $\left(p_{x}>0.05\right)$ between the groups of DR; therefore, no association with the existence of microvascular complications and also risk factors.

Conclusions. More than two-thirds of patients included in the research have some signs of DR. Because of the strong association of DR and other microvascular complications, patients with diabetes should be screened regularly for retinopathy, nephropathy, and neuropathy. And likewise, if a patient has at least one microvascular complication, he or she should be tested for the rest possible complications as well. According to data, most of the patients in this study have poor metabolic compensation; consequently, the metabolic compensation screening should be done certainly every three months.
\end{abstract}

Keywords: diabetic retinopathy, metabolic compensation, glycated hemoglobin, type 1 diabetes mellitus, nephropathy, neuropathy, atherosclerosis, arterial hypertension, coronary heart disease, dyslipidemia

\section{INTRODUCTION}

Diabetic retinopathy (DR) is a severe complication of diabetes mellitus (DM) that can cause vision loss to people at their working age. More than $90 \%$ of patients with type 1 diabetes mellitus (TIDM) will develop DR by 20 years post diagnosis (Leslie R.Dye, 2018). DR is more likely to develop in patients with TIDM (Kanski's Clinical Ophthalmology, 2015). Other microvascular complications coexist with DR such as diabetic neuropathy and especially diabetic nephropathy (Leslie 
R.Dye et al., 2018). From about 285 million of inhabitants who suffer from DM, one third have first signs of DR; two-thirds have vision-threatening DR including macular oedema. The leading role in the pathogenesis of DR is AH (arterial hypertension) and hyperglycemia which develops from longstanding inflammation and oxidative stress in tissues (Ryan Lee et al., 2015).

The combination of these factors leads to gradually progressive changes in retinal microvasculature causing mal-bloodied or even ischemic areas in the retina and, elevated permeability, which leads to exudation in the retina or further - macular oedema. Regarding ischemic regions in the retina, new vasculature starts to develop. From this point, proliferative diabetic retinopathy (PDR) develops which can lead to irreversible vision loss if it is not detected and treated as early as possible (Shlomo Melmed et al., 2016.). This complication can be very serious in terms of the ability to see. Sometimes vitrectomy plays a vital role in the management of severe complications of diabetic retinopathy at its end-stage. The major indications are non-clearing vitreous haemorrhage, macularinvolving or macular-threatening traction retinal detachment, and combined traction-rhegmatogenous retinal detachment (Myron Yanoff et al., 2020). Although nowadays there are many therapeutic options, the main goal is to prevent complications from occurring, that is why we are concentrating on variable disease factors and how to use them for a more extended period without complications.

\section{AIM OF THE STUDY}

To prove the development severity of diabetic retinopathy that depends on the duration of diabetes and metabolic compensation. Also, to determine retinopathy's association with other micro and macrovascular DM complications for a better understanding of what are the contributory factors for these complications to develop and which of those may coexist.

\section{MATERIALS AND METHODS}

In a retrospective study held at the Pauls Stradins Clinical University Hospital (Riga, Latvia) from January 2016 to March 2018, 79 (158 eyes) patient histories were analyzed who have type 1 diabetes mellitus. To obtain more precise research results, almost all patients have done check-up visits to one certain ophthalmologist. The IBM SPSS Statistics version 25.0 was used to process data. Tables were made in the SPSS and Microsoft Excel 2016 programs. For distribution probability Kolomogorov-Smirnov and Shaipro-Wilk test was used. Chi-Square test was used to determine difference and Cramer $V$ measure was applied for association between groups. Also, Mann-Whitney an Kruskal-Walis tests were used. To describe strength of association, eta $(\eta)$ was used. Statistically significant value $(p)$ was set at $<0.05$ or $5.00 \times 10^{-2}$.

\section{RESULTS}

Altogether 79 patient histories were analysed from which 38 (48.10\%) were men, and 41 (51.90\%) were women. The mean age of 79 patients was $40.01 \pm 14.10$ years with the interval from 19.00 to 76.00 years of age (Fig. 1). This range was not in normal distribution (median $=37.0)$. Out of 78 patients the mean duration of diabetes was $21.77 \pm 12.08$ years with an interval of less than 1.00 year to 49.00 years of the disease (Fig. 2). This range is in normal distribution (median $=20)$. The mean value of metabolic compensation of diabetes was $9.55 \% \pm 1.82 \%$, with intervals of $5.50 \%$ to $14.70 \%$. This range was not in normal distribution $($ median $=9.30)$ (Fig. 3).

DR was detected in most of the patients -61 or $79.22 \%$. Analysing this group further, we have concluded that 23 or $29.11 \%$ from all the patients have (PDR) (Fig. 4), whereas 38 or $48.10 \%$ - non-proliferative diabetic retinopathy NPDR (Fig. 5). For the smallest part - 16 or $20.25 \%$ patients DR were not detected (Fig. 6). Two patients or $2.53 \%$ did not have information whether they have or do not have DR, so they were excluded from further study.

No statistically significant difference can be detected in the mean indicator of the duration of disease; however, the duration of DM is considerably smaller in a group without DR (11.24, median $=8.50)$. Analysing 2 groups separately and using Mann-Whitney test, no statistically significant difference in the mean duration of the disease is seen $(p>0,05, \eta=0.02)$. Analysing between 3 groups, it is evident that the duration of diabetes in group without DR is significantly smaller and the difference is statistically significant $(p<0.05)$ and association is moderately tight $(\eta=0.46)$, as indicated in Fig. 7.

Metabolic compensation (\%) in diabetes mellitus is not statistically different between patients with diverse forms of DR, so no association was found $(p>0.05)$. No statistically significant difference has been detected comparing visual acuity for the right and left eye among patients with various forms of DR. However, when comparing the three groups (both types of DR and no DR), in patients without DR the visual acuity was higher in both eyes: $0.83 \pm 0.27$ dioptres. Difference between the groups is statistically significant $(p<0.05)$ for both eyes. Analysing association between DR and VA (visual acuity) it was detected that for the right eye it was higher or more precise also it was moderately tight $(\eta=0.45)$, but for the left eye - weak or eta was 0.34 (Fig. 8). From all patients who had information whether they have or not have arterial hypertension, altogether 77 , the presence of this disease was noticed in 24 or $31.17 \%$ of patients. 53 or $68.83 \%$ did not have the condition. Analysing further the form or existence of DR with the presence of $\mathrm{AH}$, it was found out that there is no statistically credible difference $(p>0.05)$ between the groups and no statistically significant association either. Also, another 
microvascular complication was analysed including nephropathy (2 patients or $2.60 \%$ ), polyneuropathy (25 or $32.47 \%)$, or both forms together (22 or $29.87 \%)$. One group without any other coexisting microvascular complications was set (27 or $35.06 \%$ ). Between DR and microvascular complications strong association $(p<0.05, \mathrm{~V}=0.61)$ with the existence of DR and even stronger association $(p<0.05, \mathrm{~V}=0.40)$ was found with the forms of DR. So we can see that in patients with diabetic non-prolipherative retinopathy (DNR) more often polyneuropathy or combination of both microvascular combination appears. Diabetic prolipherative retinopathy (DPR) patients does not have other microvascular complications whether have both complications at the same time. Furthermore, nephropathy separately was seen only in patients with DPR or without DR (Fig. 9).

Most of the patients - 59 or $76.62 \%$ - did not have any of macrovascular complications (atherosclerosis, dyslipidemia, or coronary artery disease). Only four patients had some of the macrovascular complications: three had atherosclerosis and coronary artery disease, whereas one had dyslipidemia and coronary artery disease. Further analysis revealed that there was no statistical significance $(p>0.05)$ between the groups and thereby no significant association was made between the groups of DR and macrovascular complications.

\section{DISCUSSION}

No statistically significant difference can be detected in the mean indicator of duration of the disease because in these two groups the mean indicator of duration is very similar. In this particular case, we cannot unequivocally prove the previously mentioned fact that "longer duration of diabetes means more severe DR" by Shlomo Melmed et al., 2016. Probably to prove the fact, more extensive research should be carried out comparing each patient's duration of disease with the type of DR and its stage/severity with a greater amount of patients. If we compare the three groups (both types of DR and no DR), it can be seen that the duration of DM in patients without DR is much shorter. This data corresponds with the previously mentioned facts that after 20 years in almost all diabetes mellitus type 1 patients some DR is seen. Regarding those patients included in research, any kind of DR stage is determined whether it is NPDR or PDR for those who have more than 20 years of diabetes. It also confirms the fact that in a group where the mean duration of diabetes is 11.24 years, no DR signs were yet detected like it was mentioned in Abdish $R$ Bhavasar, MD et al., 2017 publication. As it was stated in Shlomo Melmed et al., 2016, after ten years of diabetes, incidence of DR development risk can reach even $60 \%$ among patients with type 1 DM. This association is visualized in Figures 2 and 7 where it can be seen that most patients with a duration of DM longer than ten years also have an incidence of any kind DR - 61 or $79.22 \%$ patients. Unfortunately our research was limited, since we could not specify whether five years after diagnosis of DM most of the patients did not have any signs of DR (as confirmed in Abdish R Bhavasar, MD et al., 2017), because it is known that the first-time diagnostics of TIDM more often occurs in the early age of puberty, and patients up to 18 years of age mostly are examined by the child ophthalmologist. This data was missing in our research. Metabolic compensation a.k.a. HbAlc level is not statistically different between patients with distinct forms of DR. Also, compared to a group with patients who do not have DR, statistically reliable difference was not obtained between the mean values of HbAlc in the three groups, because the mean value of metabolic compensation in both types of DR and also no DR is almost identical. In scientific literature where the metabolic compensation's association with DR was examined, for example, in Chawla et al., 2014, it was stated that maximal values of metabolic compensation were seen in the following groups: (1) NPDR and (2) a group with no signs of DR. Temporary glycemic fluctuations do not affect this value, but it is known that retinal blood vessels are susceptible to these kind of changes, for example, hypoglycemic episodes. Therefore, more extensive research would determine how many hypoglycemic episodes each person has had. This value could be associated with the severity of DR. Presumably that HbAlc value is more likely to be useful if we investigate type 2 DM patients because in these cases glucose level does not tend to fluctuate as much as in TIDM patients. As it is said in The global diabetes community, $2018 \mathrm{HbAlc}$ is very crucial to be $<6.5 \%$, because it protects from the development of microvascular complications as diabetic retinopathy. Comparing BCVA for the right and the left eye in patients with diverse forms of DR in Fig. 6 we see that there is no statistically significant difference in the mean visual acuity. This means that in both forms of DR deteriorated visual acuity can be detected, which in both cases according to the World Health Organization visual deterioration classification (evaluating the eye with the best-corrected visual acuity) ranks close to a normal value of visual acuity (0.6-0.3). If a comparison is made between the three groups (Fig. 8; both forms with DR and no DR), it becomes evident that BCVA in patients without DR is noticeably higher in both eyes, which rank this value as normal. Probably the cause for deterioration of eyesight is one of the DR stages, but it is not unequivocally, because some of the patients might have had other eye diseases that are not detailed in this research. Analyzing the form of DR and its association with the presence or stages of $\mathrm{AH}$, no statistically significant difference ( $p$ $>0.05$ ) between the groups and no significant direct association between $\mathrm{AH}$ and stages of DR were found. $\mathrm{AH}$ is a secondary risk factor, which may worsen the development of DR by causing hypertensive vascular changes in retina, furthermore worsening the blood flow (Abdish $R$ Bhavasar, MD et al., 2017). The presence of $\mathrm{AH}$ is more important when DR is already developed. Correction of $\mathrm{AH}$ is crucial because it can reduce the diabetic nephropathy from developing, as 
it is mentioned in Europe's prospective research of diabetic complications, 2013. Examining the association between DR and microvascular complications, as seen in Fig. 9, it can be concluded that these complications have a strong association with the presence of DR and even stronger with the form of it as mentioned in the sources of literature. Many associations between microvascular complications and DR, and also with each other are mentioned in many scientific studies.

For example, in Aastha Chawla, 2016 publication it is mentioned that nephropathy was detected to 15$40 \%$ among TIDM patients. In the research herein, nephropathy was detected to 22 patients making $17.38 \%$, which is very similar. Speaking of other microvascular complication - polyneuropathy in Aastha Chawla, 2016 publication it is stated that almost half of patients have it. According to the research herein, 47 patients have this complication constituting 59.49\% and making up the majority. Consequently, this data corresponds to the data in other studies. Furthermore, in Aastha Chawla, 2016 publication it is mentioned that the risk for DR to develop is directly proportional to the duration of DM and severity of hyperglycemia. Pursuant to the research herein, majority of patients have the duration of DM 10, 15 and more years. Also, association with values of metabolic compensation is seen because the majority had poor and very poor metabolic compensation. The majority of patients were in a group that had a metabolic compensation value of more than $10 \%$ while the preferred value is $\leq 7 \%$. Analyzing if macrovascular complications/ existence of risk factors have an association with the existence of DR, we can conclude that there is no statistically significant difference between the groups and also no statistically significant association between these groups of DR and macrovascular complications. Therefore, in this case we cannot agree with Krentz et al., 2007 and Al-Wakeed et al. 2009 studies in which it is said that micro and macrovascular complications develop simultaneously. Also, no parallels have been detected with Matheus and Gomes, 2013 publication where it is stated that aggressive coronary disease developed in patients without microvascular complications. Although a patients group with macrovascular complications was very small after all.

\section{CONCLUSIONS}

More than two-thirds of patients included in the research herein have some signs of DR. Patients who do not have any signs of DR have a shorter mean duration of DM than those who have either proliferative or nonproliferative DR. The results partially correspond to the data from other scholarly sources. No statistically positive difference was found between the forms of DR and the mean values of metabolic compensation, although the association is mentioned in other scholarly sources. Comparing the two groups (both types of DR and no DR), it was detected that VA in patients without DR was higher in both eyes. No statistically significant difference and association were found between the type or existence of DR and $\mathrm{AH}$. The results of the research show that microvascular complications of diabetes mellitus have a strong association with the presence of DR and even stronger with the forms of DR. Consequently, patients with diabetes should be screened regularly for retinopathy, nephropathy, and neuropathy. Furthermore, if a patient has at least one microvascular complication, he or she should be tested for the rest possible complications as well. No significant association was found between the form/ existance of DR and macrovascular complications and the risk factor $-\mathrm{AH}$.

Conflict of interest: None 


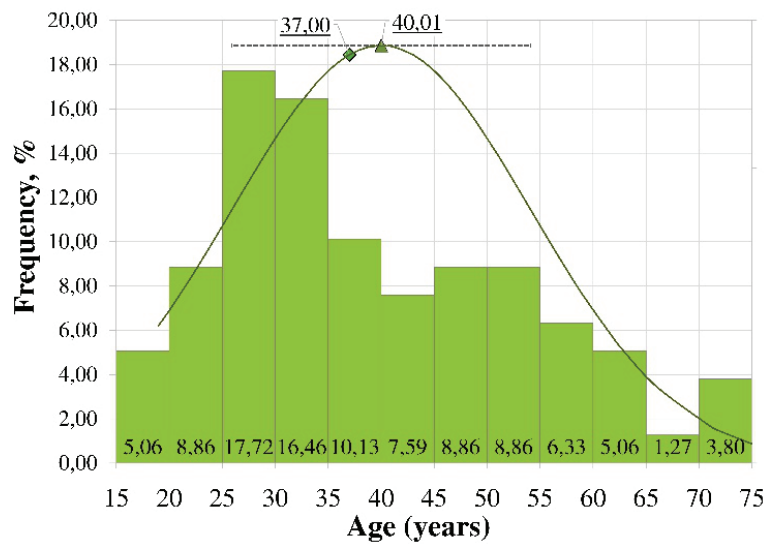

Fig. 1. Patient's age distribution in groups

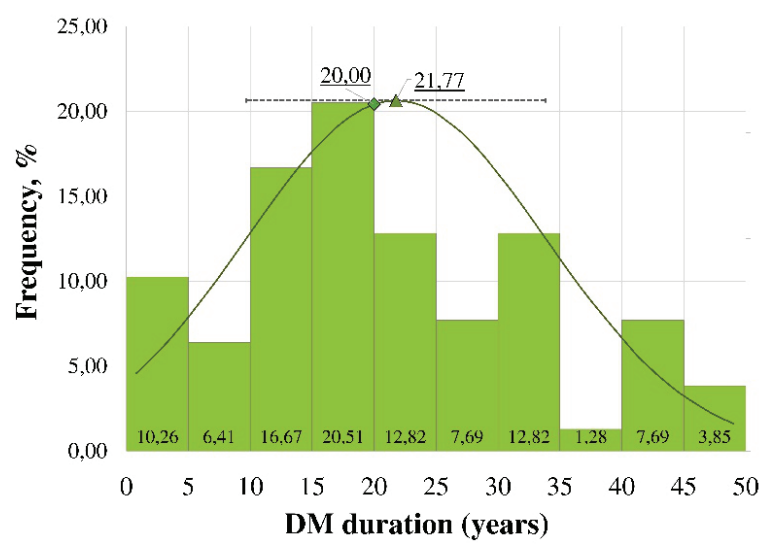

Fig. 2. Duration of diabetes mellitus distribution

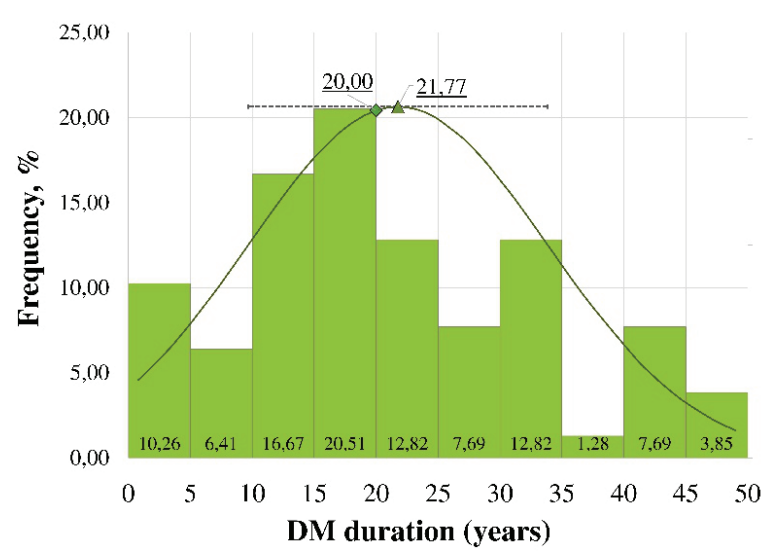

Fig. 3. Metabolic compensation values distribution

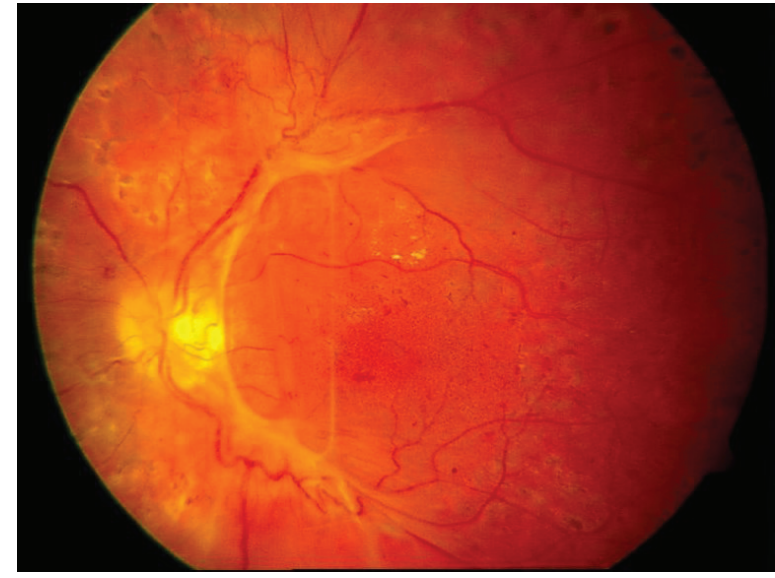

Fig. 4. Fundus photograph shows PDR with laser photocoagulation scars along blood vessel arcades. Upwards and downwards from optic disc fibrovascular membranes and underneath vascular tortuosity. Neovascularisation in the upper nasal quadrant. Grainy macular appearance, perimacular hard exudates, varioussize dot-blot haemorrhages in all quadrants

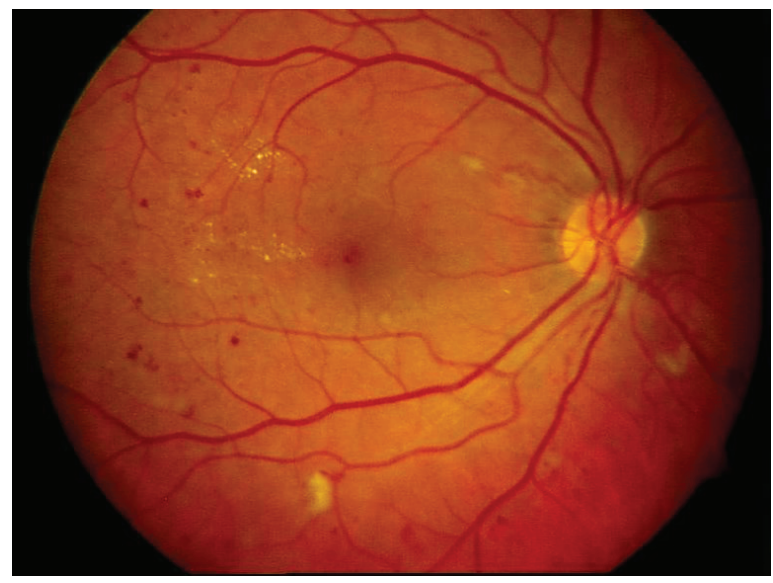

Fig. 5. Fundus photograph shows NPDR with multiple dot-blot and flame-like haemorrhages in the macula and along temporal blood vessel arcades, cotton-wool spots along inferior blood vessel arcade and groups of hard exudates temporally from the macula 


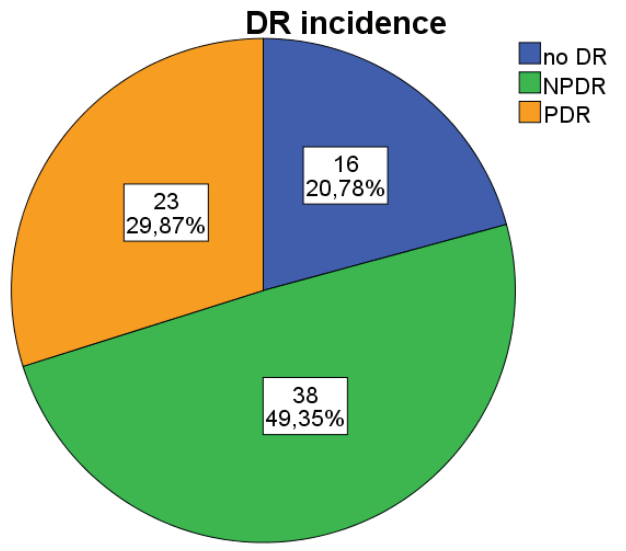

Fig. 6. Incidence of diabetic retinopathy

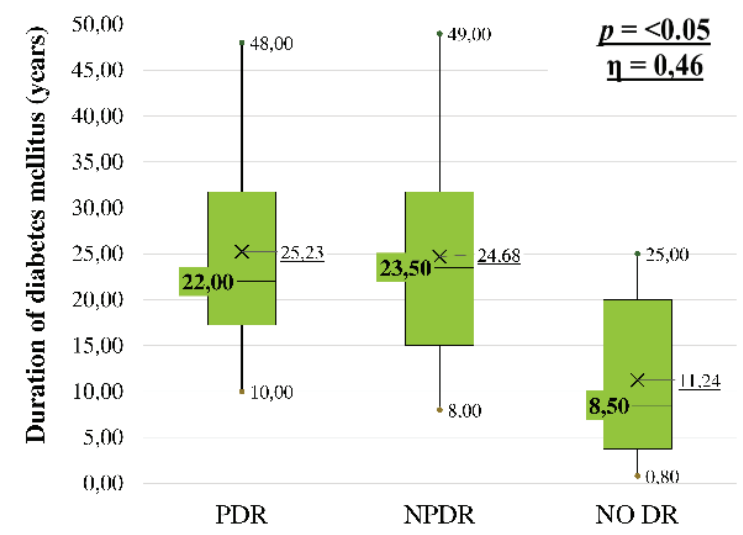

Type of diabetic retinopathy

Fig. 7 Distribution of $\mathrm{DM}$ duration (years) depending of the type of DR

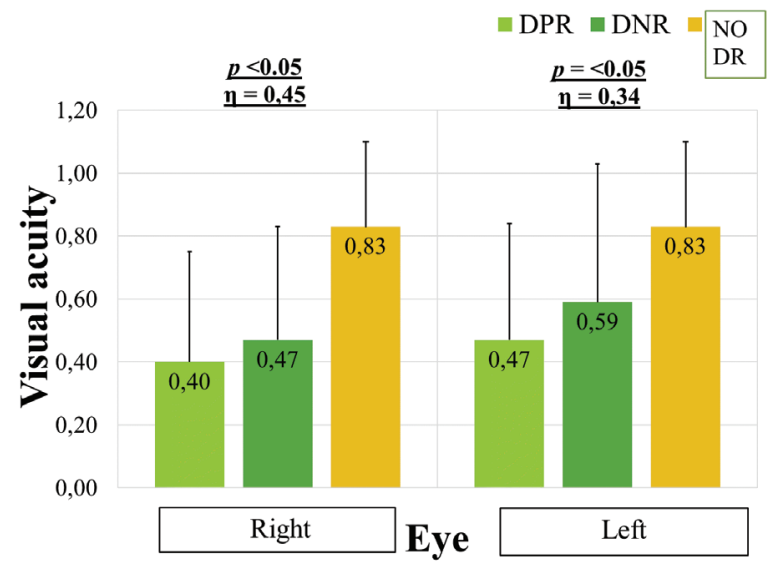

Fig. 8. Difference and association between visual acuity among different types of DR and group without DR.

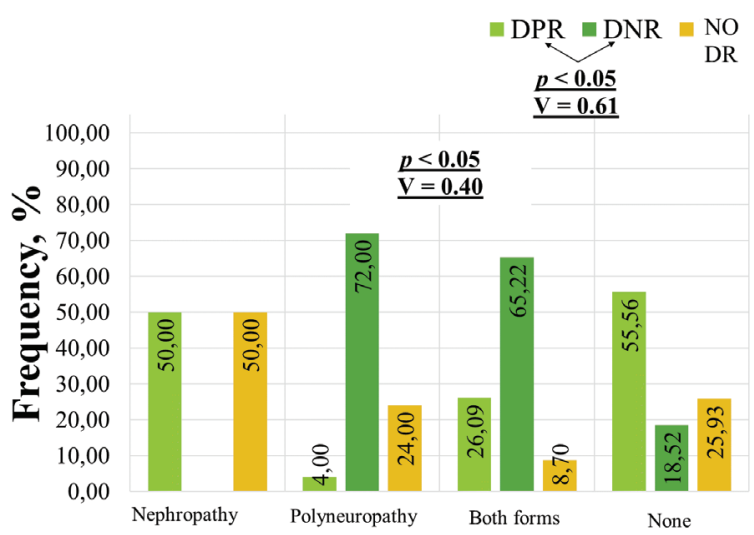

Microvascular complications

Fig. 9. DR coexistence and association with other microvascular complications among different types of DR and group without DR.

Mean visual accuity shown on the poles depending on the type of DR.

\section{REFERENCES}

1. Leslie R. Dye, MD, FACMT, FAACT, Richard K. Clise, MJ Erickson-Hogue, MD. Diabetic retinopathy, November 25, 2018, Clinical Key, Elsevier Point of Care.

2. Brad Bowling. Kanski's Clinical Ophthalmology a systemic approach, eight edition. Elsevier, 2016, p. 520 .

3. Shlomo Melmed, Kenneth S. Polonsky, P. Reed Larsen, Henry M. Kronenberg. Williams Textbook of Endocrinology, 2016, pp. 1503-1516.

4. Jack Kanski, Brad Bowling. Kanski's Clinical Ophthalmology $8^{\text {th }}$ Edition, 2015

5. Ryan Lee, Tyen Y. Wong and Chaurumathi Sabanayagam. Epidemiology of diabetic retinopathy, diabetic macular edema and related vision loss, 2015.

6. Abdish R Bhavsar, MD; Chief Editor: Romesh Khardori, MD, PhD. Diabetic Retinopathy. Updated: Apr 19, 2017. MedScape.

7. Chawla A, Chawla R, Chawla A. Correlation Between Retinopathy Microalbuminuria and Other Modifiable Risk Factors. Presented on American Diabetes Association's 75th Scientific Session; June 5-9; Boston, Massachusetts. 2015.

8. The global diabetes community. Diabetes health guidelines, 2018

9. Aastha Chawla, Rajeev Chawla and Shalini Jaggi. Microvascular and macrovascular complications in diabetes mellitus: Distinct or continuum? Jul-Aug 2016.

10. Krentz AJ, Clough G, Byrne CD. Interactions between microvascular and macrovascular disease in diabetes: pathophysiology and therapeutic implications. PubMed, 2007. 
11. Alwakeel JS, Al-Suwaida A, Isnani AC, Al-Harbi A, Alam A. Concomitant macro and microvascular cimplications in diabetic nephropathy. PubMed, 2009.

12. Matheus AS, Tannus LR, Cobas RA, Palma CC, Negrato CA, Gomes MB. Impact of diabetes on cardiovascular disease: an update. PubMed.

13. Myron Yanoff, Jay S. Duker. Ophthalmology 5th edition. Elsevier, pp. 543-552.

\section{Address:}

Lelde Ullase,

Paula Stradiña Klīniskā Universitātes slimnīca,

oftalmoloǵijas nodaļa,

Pilsonu iela 13,

Zemgales piepilsēta,

Rīga, Latvija, LV-1002.

E-pasts: ullase91@gmail.lv 